\title{
ESTUDIO DE LA ACTIVIDAD ANTIMICROBIANA DE LA CUPRITA SINTETIZADA POR RUTA QUÍMICA
}

\author{
Orfelinda Ávalo Cortez ${ }^{* a}$, David Pedro Martínez Aguilar ${ }^{\mathrm{a}}$
}

\begin{abstract}
RESUMEN
Con el auge de la nanotecnología se han desarrollado nanomateriales inorgánicos, que presentan un marcado efecto microbicida sobre una amplia variedad de microorganismos, tales como virus, bacterias y hongos. En el presente trabajo se realizó la síntesis, caracterización y estudio de la actividad antimicrobiana del óxido de cobre (I), cuprita, $\mathrm{Cu}_{2} \mathrm{O}$, obtenida por ruta química. Para la obtención de la cuprita se utilizó como precursor el sulfato de cobre pentahidratado y como reductores orgánicos el ácido ascórbico y la glucosa. La caracterización se realizó por Difracción de Rayos-X (DRX) y Microscopía Electrónica de Barrido (MEB). También se determinó la actividad antimicrobiana in vitro de las partículas de cuprita sobre la bacteria Staphylococcus aureus, determinándose la Concentración Mínima Inhibitoria(CIM). Los resultados del análisis por Difracción de Rayos X, confirmaron que a partir de la síntesis empleando ácido ascórbico como reductor y a $\mathrm{pH}=1,66$ se obtuvo $100 \%$ $\mathrm{Cu}$ puro con tamaños de partícula en el rango micrométrico y de forma poliédrica. La síntesis, empleando glucosa como reductor, dio como resultado la obtención de $100 \%$ Cuprita $\left(\mathrm{Cu}_{2} \mathrm{O}\right)$ con morfología variable, se observaron esferas, cubos y tetraedros muy dependientes de la concentración de $\mathrm{NaOH}$ empleada en cada síntesis, con tamaños de partícula en los rangos nanométrico y micrométrico, es decir se obtuvo nanopartículas y nanoestructuras de $\mathrm{Cu}_{2} \mathrm{O}$. De acuerdo a los resultados de la actividad antimicrobiana se puede concluir que el óxido de cobre $\mathrm{Cu}_{2} \mathrm{O}$ tiene efecto antimicrobiano sobre la bacteria Staphylococcus aureus, siendo la Concentración Mínima Inhibitoria, CIM, $16 \mathrm{mg} / \mathrm{mL}$.
\end{abstract}

Palabras clave: cuprita, antimicrobiana, Staphylococcus aureus.

\section{STUDY OF THE ANTIMICROBIAL ACTIVITY OF THE CUPRITA SYNTHETIZED BY CHEMICAL ROUTE}

\begin{abstract}
With the rise of nanotechnology, inorganic nanomaterials have been developed, which have a marked microbicide effect on a wide variety of microorganisms, such as viruses, bacteria and fungi. In the present work, the synthesis, characterization and study of the antimicrobial

\footnotetext{
a Facultad de Ingeniería Geológica, Minera y Metalúrgica, Universidad Nacional de Ingeniería, Av.
} Túpac Amaru 210, Lima 25, Perú, oavalo@uni.edu.pe
\end{abstract}


activity of copper oxide (I), cuprite, $\mathrm{Cu}_{2} \mathrm{O}$, obtained by chemical route was carried out. Copper sulfate pentahydrate was used as the precursor ascorbic acid and glucose, were used as organic reducers. The characterization was carried out by X-ray diffraction (XRD) and scanning electron microscopy (SEM). The in vitro antimicrobial activity of the cuprite particles on the Staphylococcus aureus bacterium was also determined, determining the Minimum Inhibitory Concentration (MIC). The results of the analysis by X-ray diffraction, confirmed that from the synthesis using ascorbic acid as a reducer and at $\mathrm{pH}=1.66,100 \%$ pure $\mathrm{Cu}$ was obtained with particle sizes in the micrometric range and polyhedral shape. The synthesis using glucose as a reducer resulted in the obtaining of $100 \%$ Cuprite $\left(\mathrm{Cu}_{2} \mathrm{O}\right)$ with variable morphology, spheres, cubes and tetrahedra were observed, very dependent on the concentration of $\mathrm{NaOH}$ used in each synthesis, with particle sizes in the nanoscale and micrometer, that is, nanoparticles and $\mathrm{Cu}_{2} \mathrm{O}$ nanostructures were obtained. According to the results of the antimicrobial activity, it can be concluded that $\mathrm{Cu}_{2} \mathrm{O}$ copper oxide has an antimicrobial effect on Staphylococcus aureus bacteria, with the MIC of $16 \mathrm{mg} / \mathrm{Ml}$.

Key words: cuprite, antimicrobial, Staphylococcus aureus.

\section{INTRODUCCIÓN}

El Staphylococcus aureus es una bacteria gram positiva que se encuentra ampliamente diseminada en el ambiente ya que posee características particulares de virulencia y resistencia contra antibióticos, lo cual representa un grave problema de salud, además esta bacteria tiene características genéticas que le han permitido convertirse en una de las bacterias más importantes en los hospitales y en las enfermedades transmitidas por alimentos ${ }^{1}$. De acuerdo a la literatura se sabe que el cobre y los óxidos de cobre son utilizados en diversos equipos sanitarios y médicos por sus propiedades bactericidas y antimicrobianas ${ }^{2}$. El mecanismo que explica la actividad antibacteriana es la capacidad del cobre para ceder y aceptar electrones en forma continua. Los iones de cobre se desprenden de superficies de cobre penetrando en la célula bacteriana produciendo la ruptura de la membrana citoplasmática que favorece la entrada de iones cobre, lo que conduce a una disfunción de la membrana y a un aumento del estrés oxidativo. A nivel citoplasmático hay una alteración de la síntesis proteica y daño funcional de enzimas esenciales produciéndose la muerte celular y degradación del ADN bacteriano $^{3}$. Si bien el cobre ocasiona que la bacteria pierda su capacidad de replicarse al alterar su estructura molecular, se sabe que no genera mutaciones en el ADN. Cualquiera de estos mecanismos afecta la supervivencia de las bacterias, pero dependerá de la concentración del metal para ver el efecto bacteriostático o bactericida. La Concentración Mínima Inhibitoria (CIM), en microbiología, es la concentración más baja de un antimicrobiano que inhibe el crecimiento de un microorganismo después de su incubación. La concentración inhibitoria mínima es importante en diagnósticos de laboratorio para confirmar la resistencia de microorganismos a un agente antimicrobiano y además para monitorizar la actividad de los nuevos agentes antimicrobianos ${ }^{4}$. 
En la literatura existen diversos trabajos sobre síntesis de cobre, $\mathrm{CuO}$ y $\mathrm{Cu}_{2} \mathrm{O}$, empleando reductores orgánicos y surfactantes cuyos resultados muestran que la síntesis por esta ruta química es sencilla y, lo más importante, permite el control del tamaño y forma de las partículas sintetizadas ${ }^{5-10}$. Existen también publicaciones respecto a la actividad antimicrobiana del cobre y de los óxidos de cobre sobre diversas bacterias tanto gram positivas como gram negativas, que en general sugieren que las nanopartículas de $\mathrm{Cu}, \mathrm{CuO}$ y $\mathrm{Cu}_{2} \mathrm{O}$ pueden ser consideradas como nuevos agentes efectivos de bacterias resistentes a múltiples fármacos ${ }^{4,11-17}$. El presente trabajo de investigación tiene como objetivo sintetizar la cuprita, $\mathrm{Cu}_{2} \mathrm{O}$, a partir de la sal precursora $\mathrm{CuSO}_{4} \cdot 5 \mathrm{H}_{2} \mathrm{O}$ y empleando como reductores orgánicos el ácido ascórbico, la glucosa y la hidracina, aprovechando la ventaja de que el empleo de reductores orgánicos requiere bajas temperaturas durante el procesos de síntesis. Realizar la caracterización microestructural de las partículas sintetizadas a través de Análisis por Difracción de Rayos-X (DRX) y Microscopia Electrónica de Barrido (MEB) y finalmente estudiar la actividad microbicida de la cuprita sobre la bacteria Staphylococcus aureus a fin de determinar la Concentración Inhibitoria Mínima (CIM).

\section{PARTE EXPERIMENTAL}

Para la síntesis del óxido cobre se partió del precursor en forma de sulfato de cobre pentahidratado, $\left(\mathrm{CuSO}_{4} .5 \mathrm{H}_{2} \mathrm{O}\right)$, el cual fue reducido con ácido ascórbico $\left(\mathrm{C}_{6} \mathrm{H}_{8} \mathrm{O}_{6}\right)$ en medio ácido y con glucosa $\left(\mathrm{C}_{6} \mathrm{H}_{12} \mathrm{O}_{6}\right)$ en medio básico.

\section{Síntesis empleando ácido ascórbico como reductor}

Se procedió a pesar 5,03g de sulfato de cobre pentahidratado y 12,01g de ácido ascórbico. Luego se llevaron a disolución la sal precursora y el reductor orgánico a temperatura ambiente, empleando agua destilada. Se agregó $18 \mathrm{~mL}$ de agua destilada a los $5,3 \mathrm{~g}$ de $\mathrm{CuSO}_{4} * 5 \mathrm{H}_{2} \mathrm{O}$ y $30 \mathrm{~mL}$ de agua destilada a los 12,01g de ácido ascórbico. Luego de la disolución de las sales por separado, se procedió a adicionar de a poco la solución que contenía el sulfato de cobre pentahidratado sobre el recipiente que contenía la solución de ácido ascórbico, agitando vigorosamente. Se observó la formación de una solución final de color verde oscuro con un pH de aproximadamente 1,66. Luego de finalizar la adición de toda la solución de sulfato de cobre pentahidratado a la solución de ácido ascórbico, la solución final fue calentada a una temperatura de $70^{\circ} \mathrm{C}$ y por un tiempo de $20 \mathrm{~min}$, empleando una plancha de calentamiento con agitación magnética. Se observó la formación de un precipitado de color naranja en la base del vaso, que nos daba indicios que se trataba de cobre puro. Se procedió a decantar la solución sobrenadante y a lavar el precipitado varias veces con agua destilada. El lavado final se realizó con alcohol, se procedió al secado de la muestra, el envasado y etiquetado

\section{Síntesis empleando glucosa como reductor}

Se pesó $5,71 \mathrm{~g}$ de sulfato de cobre pentahidratado y se enrasó a $50 \mathrm{ml}$ en un vaso de precipitado con agua destilada. Se pesaron $2,13 \mathrm{~g}$ de glucosa y 3,78g de hidróxido de sodio, enrasándose cada uno a $25 \mathrm{ml}$ en sus respectivos vasos de precipitado. 
Inicialmente, se agregó el hidróxido de sodio al vaso que contenía la glucosa. Se observó la formación de una solución de un tono ligeramente amarillento. A esta solución amarillenta le agregamos poco a poco el sulfato de cobre pentahidratado que estaba colocado en una bureta. A medida que se adicionaba el $\mathrm{CuSO}_{4} .5 \mathrm{H}_{2} \mathrm{O}$ este cambiaba de una tonalidad azul a verde oscuro. Se fue agitando constantemente la solución durante todo el proceso y se anotaba el cambio de $\mathrm{pH}$ en cada adición de sulfato de cobre de la bureta. Conforme aumentaba el $\mathrm{pH}$, se observaba que la solución se volvía más oscura, hasta que al llegar a un $\mathrm{pH}$ de 12,3 se observó un precipitado rojizo. Finalizada la titulación, se procedió a calentar el vaso que contenía al precipitado en una plancha de calentamiento a $60^{\circ} \mathrm{C}$ durante 30 minutos. Luego se procedió a decantar para desechar la solución sobrenadante y luego se procede a lavar el precipitado cinco veces con agua destilada y una última lavada con alcohol. Finalmente, se procedió a secar el precipitado a menos de $50^{\circ} \mathrm{C}$. Una vez seca la muestra se procedió a determinar el peso final que fue de $1,47 \mathrm{~g}$.

Las muestras finales fueron enviadas a los laboratorios externos para su análisis por Difracción de Rayos-X (DRX) y Microscopia Electrónica de Barrido (MEB).

\section{RESULTADOS Y DISCUSIÓN}

\section{Análisis de los difractogramas DRX de la muestra sintetizada empleando ácido ascórbico como reductor en medio ácido.}

Realizando el análisis cualitativo se puede observar que los picos del difractograma de esta muestra coinciden con los picos correspondientes al cobre puro, tal como se puede observar en la figura 1. Por lo tanto se puede concluir que de la reducción del sulfato de cobre con ácido ascórbico a $\mathrm{pH}=1,66$ se obtiene $100 \%$ cobre puro, tal como fue establecido en el trabajo de Liu King Ming et al (2012), donde concluyen que a pH bien bajos se obtiene cobre puro de mayor tamaño (micrométricos) y a $\mathrm{pH}$ altos se obtiene cuprita de tamaños más pequeños (nanométricos), además establecen que el posible mecanismo de formación del cobre puro a pH mayores a 2,5 es a través de las siguientes etapas: $\mathrm{Cu}^{2+} \rightarrow \mathrm{Cu}(\mathrm{OH})_{2} \rightarrow \mathrm{Cu}_{2} \mathrm{O} \rightarrow \mathrm{Cu}$, es decir los iones $\mathrm{Cu}^{2+}$ son transformados inicialmente a $\mathrm{Cu}(\mathrm{OH})_{2}$ y luego el $\mathrm{Cu}(\mathrm{OH})_{2}$ se reduce a $\mathrm{Cu} 2 \mathrm{O}$ por el ácido ascórbico. $\mathrm{El} \mathrm{Cu}_{2} \mathrm{O}$ finalmente se reduce a partículas de $\mathrm{Cu}$, por lo tanto establecen al $\mathrm{Cu}_{2} \mathrm{O}$ como producto intermedio y representan el proceso de reducción a través de las siguientes reacciones ${ }^{18}$ :

$$
\begin{aligned}
& \mathrm{Cu}(\mathrm{OH})_{2}+\mathrm{C}_{6} \mathrm{H}_{8} \mathrm{O}_{6} \rightarrow \mathrm{Cu}_{2} \mathrm{O}+\mathrm{C}_{6} \mathrm{H}_{6} \mathrm{O}_{6}+\mathrm{H}_{2} \mathrm{O} \\
& \mathrm{Cu}_{2} \mathrm{O}+\mathrm{C}_{6} \mathrm{H}_{8} \mathrm{O}_{6} \rightarrow \mathrm{Cu}+\mathrm{C}_{6} \mathrm{H}_{6} \mathrm{O}_{6}+\mathrm{H}_{2} \mathrm{O}
\end{aligned}
$$

Para poder observar las posibles rutas del proceso de reducción del sulfato de cobre en medio acuoso, podemos emplear el Software HSC 6, para construir el Diagrama de Pourbaix del Sistema Cu-O-H a $60^{\circ} \mathrm{C}$ y para la concentración de $\mathrm{Cu}$ en la solución, que es de 1.14 molal, tal como se muestra en la figura 2. Claramente, se puede observar que para el pH igual a 1,66, que fue el empleado en la síntesis con el ácido ascórbico como reductor, se puede sintetizar cobre puro en un solo paso. 


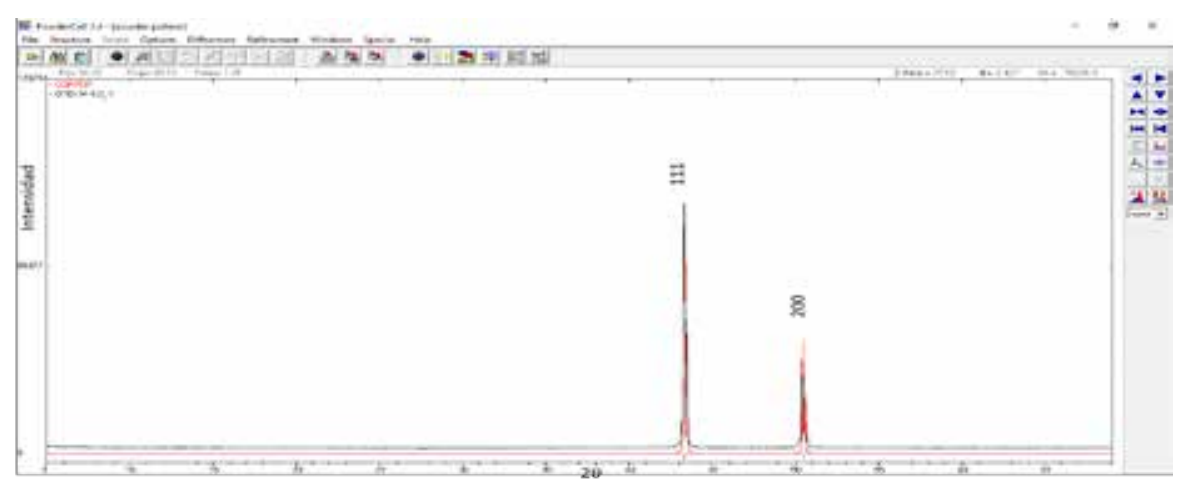

Figura 1. Análisis comparativo del difractograma patrón del cobre y el difractograma de la muestra experimental obtenida de la reducción del sulfato de cobre con ácido ascórbico.

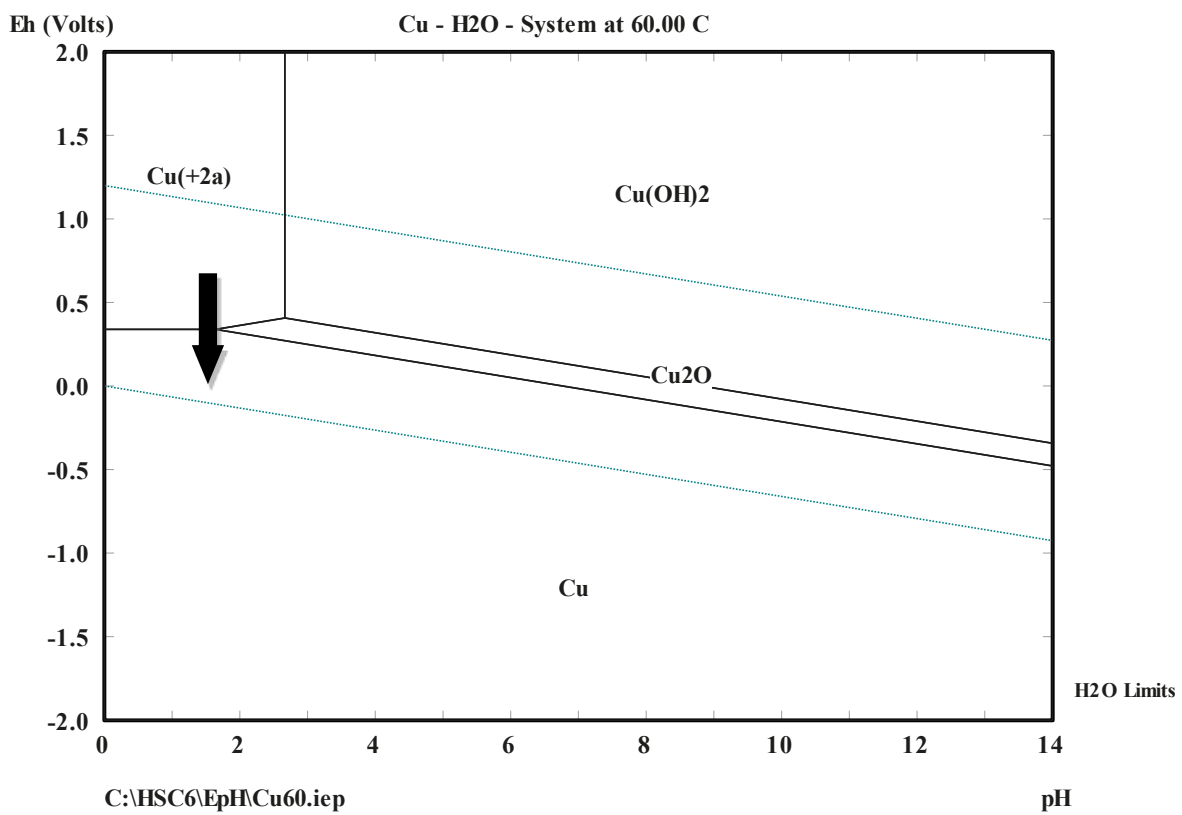

Figura 2. Diagrama de Pourbaix del Sistema Cu-O-H a $60^{\circ} \mathrm{C}$ y para una concentración de cobre 1.14 molal, levantados con el Software HSC 6.

\section{Análisis de los difractogramas DRX de la muestra sintetizada empleando glucosa como reductor en medio básico.}

En trabajos anteriores, empleando glucosa e hidracina como reductores, se encontró que, dependiendo del rango de $\mathrm{pH}$ empleado en la síntesis, es posible obtener una mezcla de metal/óxido, es decir $\mathrm{Cu} / \mathrm{Cu}_{2} \mathrm{O}$, tanto en el caso del uso de glucosa como reductor y también de la hidracina ${ }^{19}$. Al comparar los difractogramas de la muestra experimental con los patrones 
de difracción del cobre y de la cuprita, se puede observar que la muestra obtenida empleando glucosa como reductor a $\mathrm{pH}=12,3$ presenta picos de cobre y cuprita, tal como se observa en la figura 3. Haciendo el análisis cuantitativo se determinó que la muestra final contenía 93,5\% de cuprita y 6,5\% de cobre, con un tamaño de cristalito de 83,23 nm. Algunos investigadores establecieron que los iones $\mathrm{Cu}^{2+}$ se reducen primero a $\mathrm{Cu}^{+}$por la glucosa y luego el $\mathrm{Cu}^{+}$ reacciona con el $\mathrm{OH}^{-}$para formar $\mathrm{Cu}_{2} \mathrm{O}$, de acuerdo a las siguientes ecuaciones ${ }^{7}$ :

$$
\begin{aligned}
& \mathrm{Cu}(\mathrm{OH})_{2}=\mathrm{Cu}^{2+}+2 \mathrm{OH}^{-} \quad\left(\mathrm{o} \mathrm{CuO}+\mathrm{H}_{2} \mathrm{O}=\mathrm{Cu}^{2+}+2 \mathrm{OH}^{-}\right) \\
& \mathrm{Cu}^{2+}+3 \mathrm{OH}^{-}+\mathrm{C}_{6} \mathrm{H}_{12} \mathrm{O}_{6}=\mathrm{Cu}^{+}+\mathrm{C}_{6} \mathrm{H}_{11} \mathrm{O}_{7}+2 \mathrm{H}_{2} \mathrm{O} \\
& \mathrm{Cu}^{+}+\mathrm{OH}-=\mathrm{CuOH} \\
& 2 \mathrm{CuOH}=\mathrm{Cu}_{2} \mathrm{O}+\mathrm{H}_{2} \mathrm{O}
\end{aligned}
$$

De acuerdo a los resultados anteriores, se pudo deducir que la síntesis empleando a la glucosa como reductor, podría conducirnos a obtener $100 \%$ cuprita y por eso se llevó a cabo pruebas experimentales modificando el $\mathrm{pH}$, es decir variando la cantidad de $\mathrm{NaOH}$ empleada durante el proceso de síntesis de la muestra. Al hacer el análisis comparativo de los difractogramas experimental y el de la cuprita se observa que en este caso solo aparecen los picos de la cuprita, por tanto se obtuvo $100 \%$ cuprita, figura 4 .

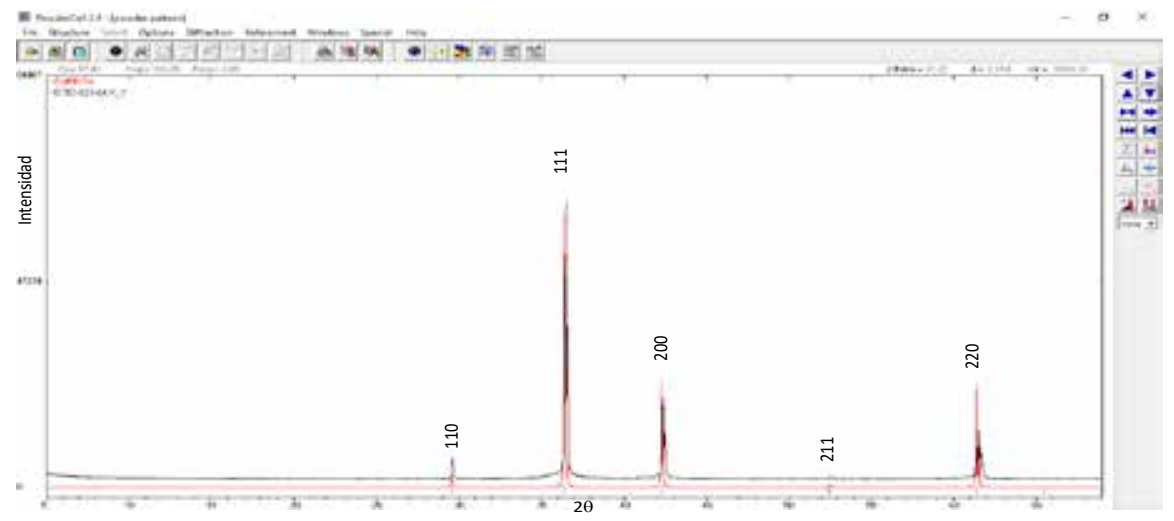

Figura 3. Análisis comparativo de los difractogramas patrón del cobre y cuprita con el difractograma de la muestra experimental obtenida de la reducción del sulfato de cobre con glucosa a $\mathrm{pH}=12,3$ 


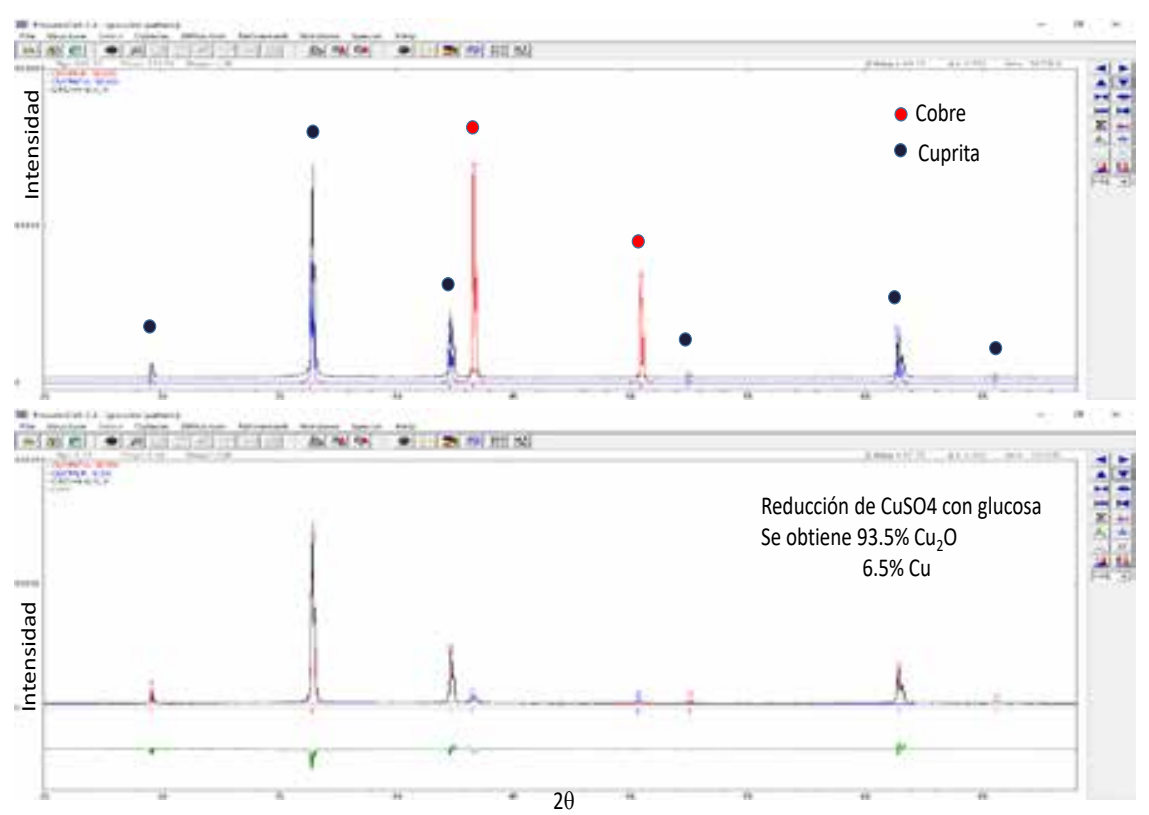

Figura 4. Análisis comparativo del ditractograma patrón de la cuprita y el ditractograma de la muestra experimental obtenida de la reducción del sulfato de cobre con glucosa a pH=12.

Otra modificación que se realizó durante el proceso de síntesis empleando la glucosa como reductor fue la de realizar la síntesis de la cuprita en frío, es decir sin calentamiento. Al realizar el análisis comparativo del difractograma experimental con el difractograma patrón de la cuprita, se puede observar que se obtuvo $100 \%$ cuprita, tal como se puede observar en la figura 5.

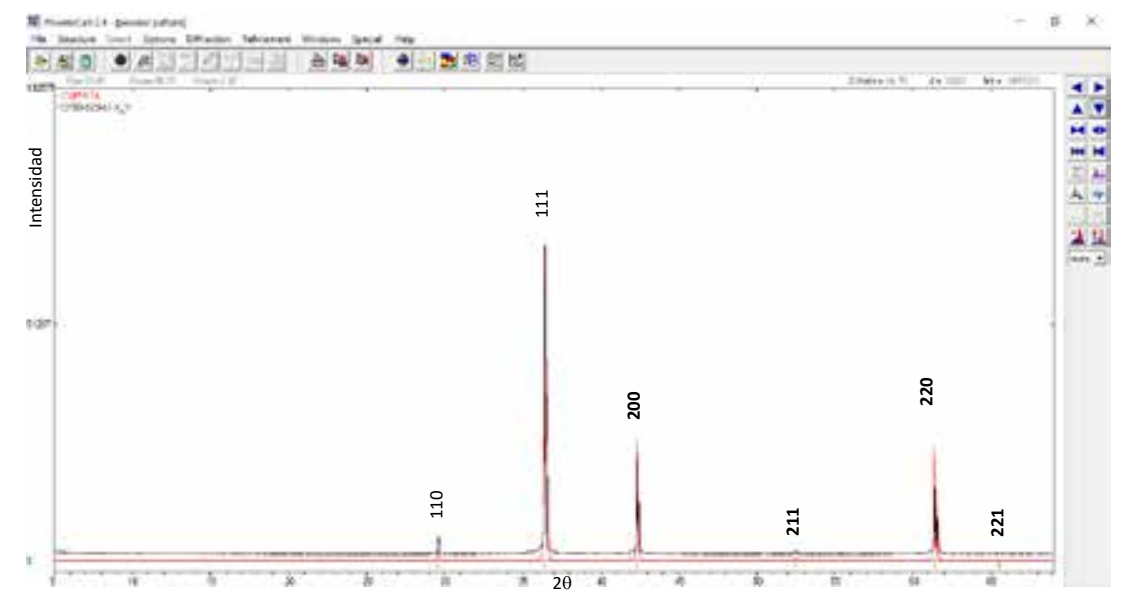

Figura 5. Análisis comparativo del difractograma patrón de la cuprita y el difractograma de la muestra experimental obtenida de la reducción del sulfato de cobre con glucosa a $\mathrm{pH}=12,3$, síntesis en frío. 


\section{Análisis de las micrografías MEB de la muestra de cobre obtenida empleando como reductor el ácido ascórbico en medio ácido.}

La muestra de cobre fue enviada al Laboratorio de Microscopia Electrónica de Barrido, de la Facultad de Ciencias de la Universidad Nacional de Ingeniería. Se puede observar en la figura 6 que las partículas están aglomeradas y que presentan una distribución de tamaños casi homogénea, mostrando cristales facetados.

En la figura 7, con una magnificación mayor, se puede observar claramente que los cristales de cobre puro son partículas poliédricas micrométricas de entre 3 - $6 \mu \mathrm{m}$ de tamaño promedio. Esto concuerda con lo encontrado en la literatura, donde establece que a medida que se aumenta el $\mathrm{pH}$ el tamaño de la partícula tiende a disminuir, en nuestro caso el $\mathrm{pH}$ experimental fue de 1,66 , que es un valor muy bajo y por tanto se obtiene partículas de mayor tamaño.

Es decir, a partir de la reducción del sulfato de cobre empleando ácido ascórbico y a un $\mathrm{pH}$ de 1,66 se consiguen obtener partículas en forma de poliedros de tamaños micrométricos.

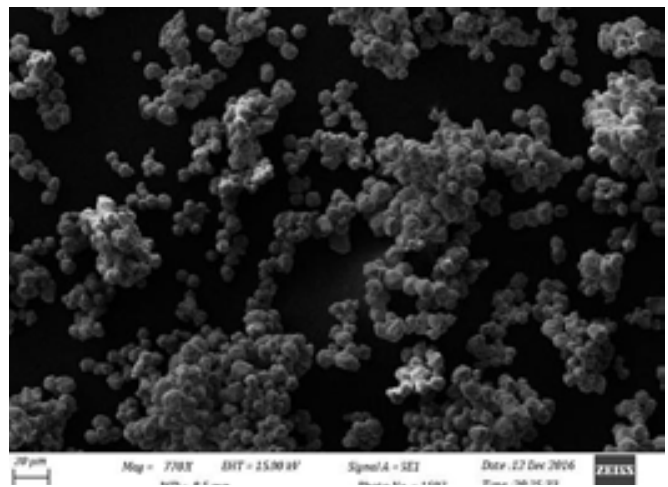

Figura 6. Micrografías MEB a una magnificación de $770 \mathrm{X}$ de la muestra obtenidaempleando como reductor el ácido ascórbico en medio ácido $(\mathrm{pH}=1,66)$.

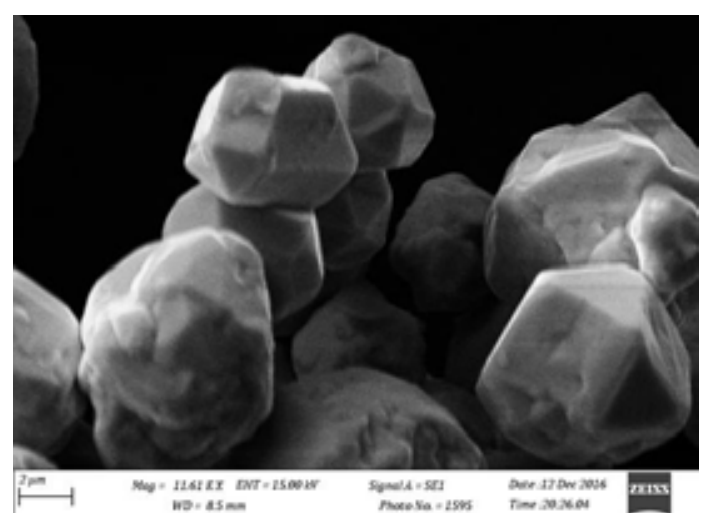

Figura 7. Micrografías MEB 11.61 KX de la muestra de cobre obtenida a partir del precursor sulfato de cobre y ácido ascórbico como reductor. 
Análisis de las micrografías $\mathrm{MEB}$ de la cuprita sintetizada con glucosa a $\mathrm{pH}=12$ y con calentamiento

En la figura 8 se presenta la micrografía MEB de la muestra de cuprita sintetizada con glucosa a pH igual a 12 y con calentamiento. Se puede observar que la morfología de las partículas es esférica y con una distribución de tamaños heterogéneos. Estas micrografías MEB fueron obtenidas en el Microscopio Electrónico de Barrido tipo Tapletop de la PUC-Rio.

Esta misma muestra fue analizada en el Microscopio Electrónico de Barrido de la Facultad de Ciencias de la UNI con la finalidad de tener micrografías con mayor magnificación. En la figura 9 se puede observar que a una magnificación de $112.20 \mathrm{KX}$ se logra observar que se logran sintetizar partículas de cuprita de tamaño nanométrico, es decir nanopartículas. Lamentablemente, no se tuvo acceso a un Microscopio Electrónico de Transmisión (MET) para lograr mayor magnificación y tener más detalle de las muestras.

\section{Análisis de las micrografías $\mathrm{MEB}$ de la cuprita sintetizada con glucosa a $\mathrm{pH}=12,3$ y con calentamiento}

En la figura 10 se presenta las micrografías MEB a $5 \mathrm{KX}, 10 \mathrm{KX}, 20 \mathrm{KX}$ y $30 \mathrm{KX}$ de la muestra de cuprita sintetizada con glucosa a $\mathrm{pH}$ igual a 12,3 y con calentamiento. Se puede observar que la morfología de las partículas es una mistura de cuasi-esféricas, cúbicas y tetraédricas con una distribución de tamaños heterogéneos. Las partículas con morfología tetraédrica son las más pequeñas con tamaño menor a 1um, las partículas cuasi-esféricas, cúbicas y algunas tetraédricas tiene un tamaño promedio de $3 \mathrm{um}$. Se puede observar en las partículas cúbicas de la micrografía a 30KX que la partícula está constituida de pequeños cristalitos. Además se puede observar que las partículas cúbicas migran a cuasi-esferas y luego estas a tetraedros.
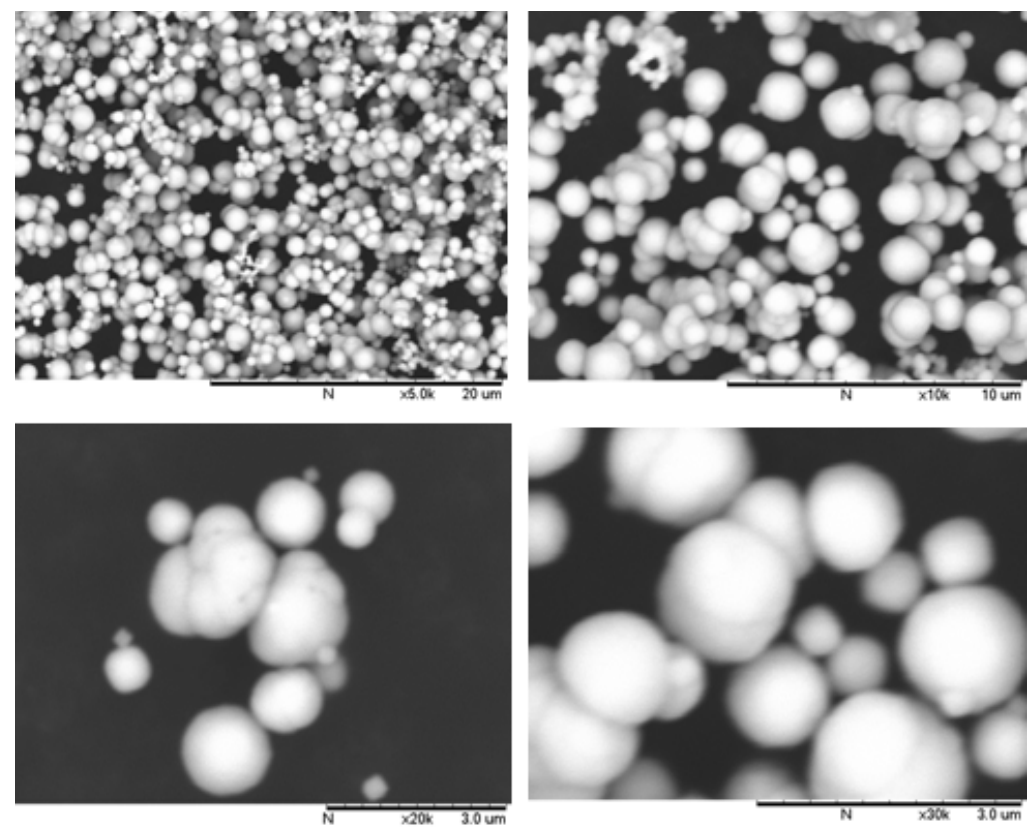

Figura 8. Micrografías MEB a $5 \mathrm{KX}, 10 \mathrm{KX}, 20 \mathrm{KX}$ y $30 \mathrm{KX}$ de la muestra de cuprita sintetizada a $\mathrm{pH}=12$ y con calentamiento. 


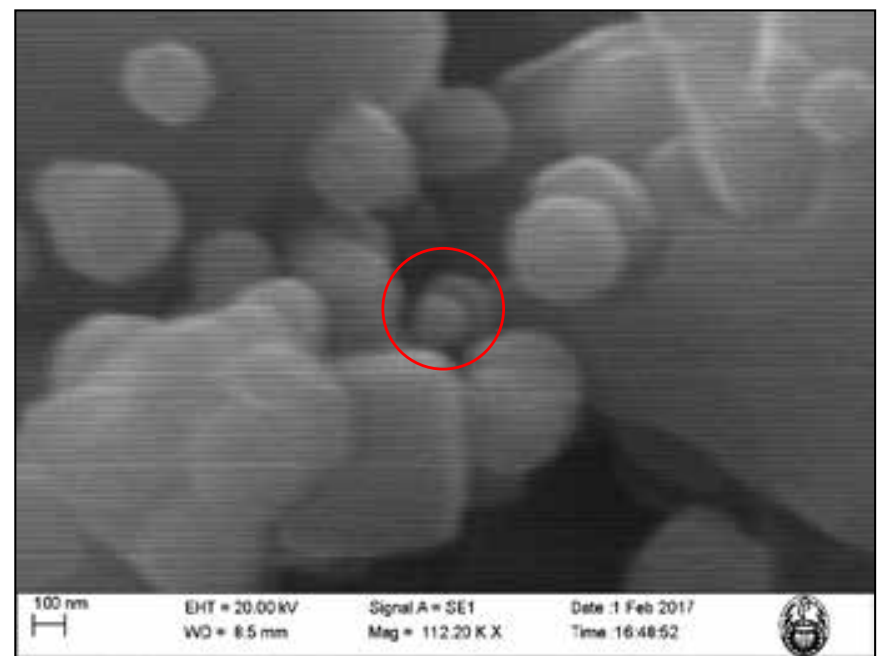

Figura 9. Micrografía MEB a $112.20 \mathrm{KX}$ de la muestra de cuprita sintetizada a $\mathrm{pH}=12$ y con calentamiento.
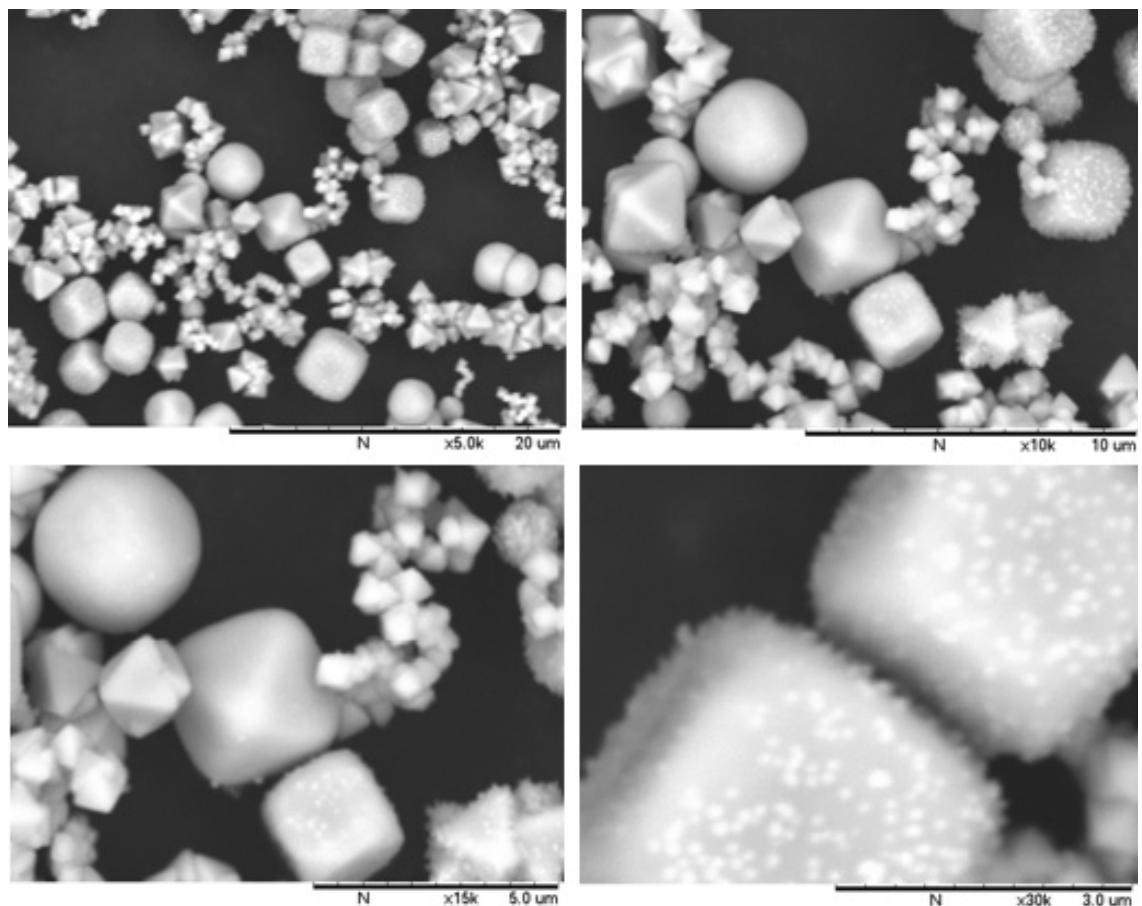

Figura 10. Micrografías MEB a $5 \mathrm{KX}, 10 \mathrm{KX}, 20 \mathrm{KX}$ y $30 \mathrm{KX}$ de la muestra de cuprita sintetizada a $\mathrm{pH}=12,3$ y con calentamiento. 


\section{Análisis de las micrografías MEB de la cuprita sintetizada con glucosa a $\mathrm{pH}=12,3$ y $\sin$ calentamiento}

En la figura 11 se presenta la micrografía MEB a $14.50 \mathrm{KX}$ de la muestra de cuprita sintetizada con glucosa a pH igual a 12,3 y sin calentamiento, es decir sintetizada en frío. Se puede observar que la morfología de las partículas es tetraédrica con una distribución de tamaño bastante homogéneo.

De acuerdo a los resultados obtenidos podemos concluir que se pueden sintetizar cuprita de diferentes morfologías reduciendo $\mathrm{Cu}$ (II) con glucosa a $60^{\circ} \mathrm{C}$ o en frío y en medio alcalino. Se encontró que la forma de las partículas de $\mathrm{Cu}_{2} \mathrm{O}$ cambia con el cambio de concentración de $\mathrm{NaOH}$, es decir con la variación del $\mathrm{pH}$. Las diferentes formas de las partículas de $\mathrm{Cu}_{2} \mathrm{O}$ se deben a la absorción de iones $\mathrm{OH}^{-}$en las partículas de $\mathrm{Cu} 2 \mathrm{O}$, que surgen en la variedad del modo de crecimiento de $\mathrm{Cu}_{2} \mathrm{O}$, y que luego influyen en la morfología final de las partículas de $\mathrm{Cu}_{2} \mathrm{O}$.

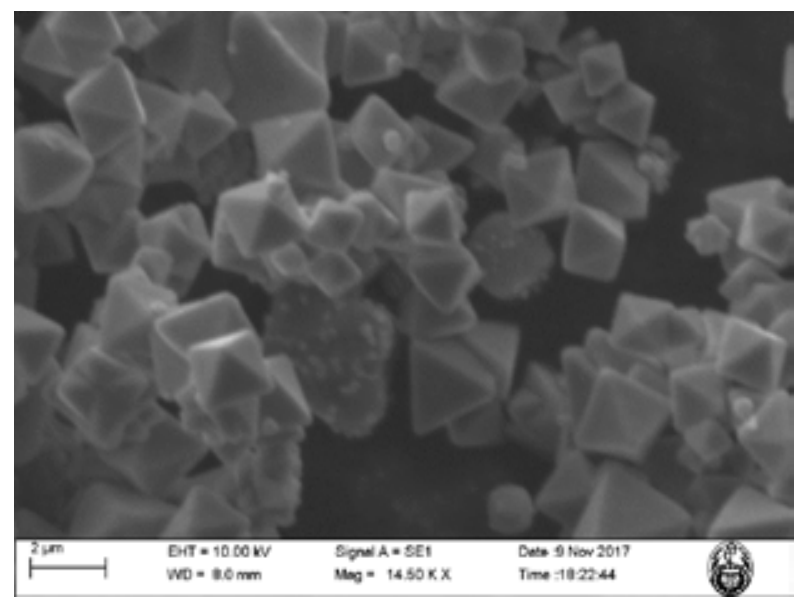

Figura 11. Micrografías MEB a 14.50 KX de la muestra de cuprita sintetizada a $\mathrm{pH}=12,3$ y sin calentamiento.

\section{Análisis de los resultados del ensayo realizado para la determinación de la Concentración Mínima Inhibitoria, CIM}

En los ensayos de Concentración Inhibitoria Mínima (CIM) se usó una cepa ATCC 43300 Staphylococcus aureus resistente a metilicina. El medio de cultivo utilizado para el crecimiento de la cepa como para la realización de la CIM fue Muller Hinton, Las partículas de cuprita fueron disueltas en este medio y la lectura del CIM se realizó por medio de espectofotometría a una longitud de onda de $600 \mathrm{~nm}$. La concentración inicial de la cepa siguió la equivalencia de 0,5 de Mac Farland.

\section{Ensayo con la muestra de cuprita (glucosa como reductor)}

En este ensayo se tomaron las concentraciones de $144,48,16,5,3,1,7$ y $0,56 \mathrm{mg} / \mathrm{mL}$ de la muestra de cuprita. La tabla 1 muestra el volumen en $\mu \mathrm{L}$ usado en la preparación de las concentraciones de cuprita. El control negativo corresponde a la concentración de $16 \mathrm{mg} / \mathrm{mL}$. 
Tabla 1. Volumen en $\mu \mathrm{L}$ usado en la preparación de las concentraciones de cuprita en el rango de concentraciones $144-0,56 \mathrm{mg} / \mathrm{mL}$

\begin{tabular}{lccccccccc}
\hline & \multirow{2}{*}{$\mathbf{B}$} & \multirow{2}{*}{$\mathbf{C}^{-}$} & \multirow{2}{*}{$\mathbf{C}^{+}$} & \multicolumn{6}{c}{ Concentración de Cobre (mg/mL) } \\
\cline { 5 - 10 } & & & $\mathbf{1 4 4}$ & $\mathbf{4 8}$ & $\mathbf{1 6}$ & $\mathbf{5 . 3}$ & $\mathbf{1 . 7}$ & $\mathbf{0 . 5 6}$ \\
\hline MH & 1500 & 1365 & 1400 & 185 & 995 & 1265 & 1355 & 1385 & 1395 \\
Cobre & & 135 & & 1215 & 405 & 135 & 45 & 15 & 5 \\
Cepa & & & 100 & 100 & 100 & 100 & 100 & 100 & 100 \\
\hline
\end{tabular}

Los resultados de la absorbancia luego de medir el OD se muestran en la tabla 2.

Tabla 2. Resultados de la absorbancia de la muestra de cuprita

\begin{tabular}{|c|c|c|c|c|c|c|c|}
\hline C- & C+ & $\begin{array}{c}144 \mathrm{mg} / \mathrm{mL} \\
\text { (1) }\end{array}$ & $\begin{array}{c}48 \mathrm{mg} / \mathrm{mL} \\
\text { (2) }\end{array}$ & $\begin{array}{c}16 \mathrm{mg} / \mathrm{mL} \\
\text { (3) }\end{array}$ & $\begin{array}{c}5.3 \mathrm{mg} / \mathrm{mL} \\
\text { (4) }\end{array}$ & $1.7 \mathrm{mg} / \mathrm{mL}$ & $\begin{array}{c}0.56 \mathrm{mg} / \mathrm{mL} \\
\text { (6) }\end{array}$ \\
\hline 1,182 & 0,65 & 2,478 & 2,154 & 1,025 & 0,521 & 0,569 & 0,554 \\
\hline 1,163 & 0,716 & 2,318 & 2,001 & 1,003 & 0,524 & 0,6 & 0,593 \\
\hline 1,082 & 0,727 & 2,272 & 2,021 & 0,978 & 0,526 & 0,654 & 0,576 \\
\hline 1,142 & 0,698 & 2,356 & 2,059 & 1,002 & 0,524 & 0,608 & 0,574 \\
\hline
\end{tabular}

En las figuras 12 y 13 se muestran la coloración luego de preparar los viales con la cepa y las concentraciones de las partículas que se mantienen hasta terminar la MIC. En la figura 12 se observa la coloración luego de vortexear el medio y en la figura 13 se observa que las nanopartículas han sedimentado luego de 20 minutos. Como se observa en el control negativo, la coloración afecta el OD. La diferencia entre el control negativo con su contraparte (vial $3=$ $16 \mathrm{mg} / \mathrm{mL}$ ) da un OD de 0,304 , por lo cual la CIM de la muestra podría ser $16 \mathrm{mg} / \mathrm{mL}$.

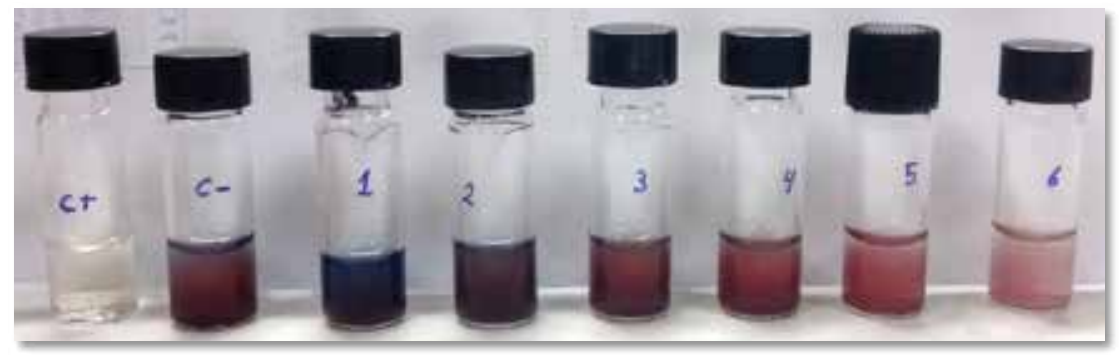

Figura 12. Imagen que muestra la coloración de los viales luego de vortexear.

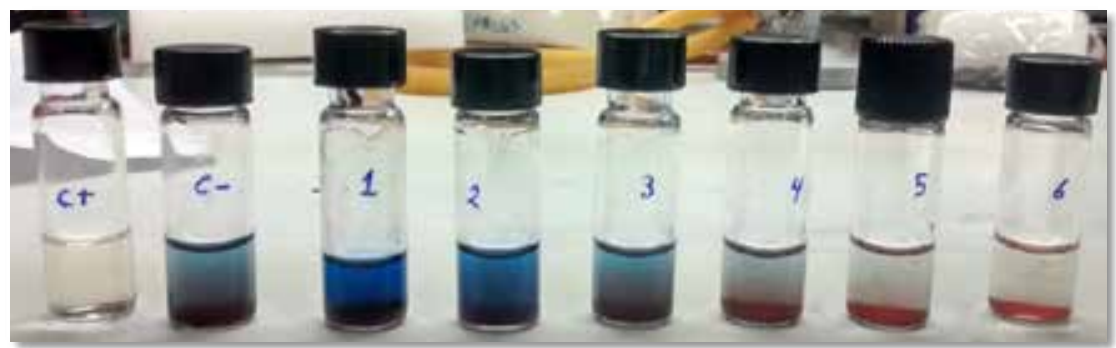

Figura 13. Imagen que muestra la sedimentación de las partículas luego de 20 min del vortexeado 


\section{Análisis de los resultados de las pruebas de crecimiento bacteriano.}

En la figura 14 se observa en la placa Petri de la parte superior, crecimiento de la cepa control (Staphylococcus aureus) en medio Manitol salado. En la placa Petri de la parte inferior izquierda se observa el crecimiento de la cepa donde la concentración del vial 4 corresponde a $5,3 \mathrm{mg} / \mathrm{mL}$, mientras que en la placa Petri de la parte inferior derecha no se observa crecimiento de la cepa a una concentración de $144 \mathrm{mg} / \mathrm{mL}$. Por tanto, la Concentración Inhibitoria Mínima, CIM corresponde a la concentración en la cual la placa que no muestra crecimiento seguida de la placa que sí mostró crecimiento y esto sería a una concentración de $16 \mathrm{mg} / \mathrm{mL}$.

Por tanto, este valor de $16 \mathrm{mg} / \mathrm{mL}$ sería la Concentración Inhibitoria Mínima, CIM, de la cuprita, necesaria para evitar el crecimiento de la bacteria Staphylococcus aureus, lográndose, finalmente, alcanzar el objetivo final del presente trabajo de investigación.

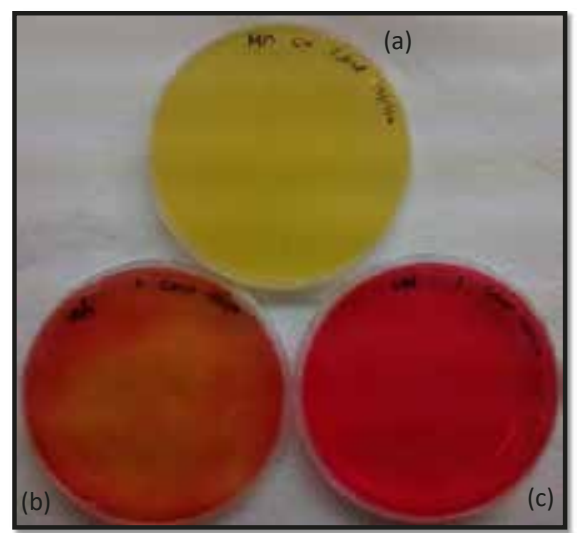

Figura 14. Imagen que muestra el crecimiento bacteriano. a) Crecimiento de la bacteria Staphylococcus aureus sin presencia de cuprita. b) Parcial crecimiento de la bacteria Staphylococcus aureus en presencia de $5,3 \mathrm{mg} / \mathrm{mL}$ de cuprita. c) Total inhibición del crecimiento de la bacteria Staphylococcus aureus en presencia de $16 \mathrm{mg} / \mathrm{mL}$ de cuprita.

\section{CONCLUSIONES}

Lo que se obtiene a partir de la síntesis de sulfato de cobre pentahidratado empleando ácido ascórbico como reductor a un $\mathrm{pH}$ igual a 1,66 es $100 \% \mathrm{Cu}$ puro, con una morfología poliédrica y de tamaño micrométrico, mientras que de la síntesis de sulfato de cobre pentahidratado empleando glucosa como reductor y a un $\mathrm{pH}$ de 12,3 se obtiene 93,5\% de $\mathrm{Cu} 2 \mathrm{O}$ y 6,5 $\%$ de $\mathrm{Cu}$. Las partículas obtenidas en estas condiciones tienen formas de esferas, cubos y tetraedros, de tamaño heterogéneo.

Se puede obtener $100 \%$ cuprita modificando la concentración del $\mathrm{NaOH}$ durante la síntesis que afecta tanto al $\mathrm{pH}$, a la morfología y al tamaño de las partículas. $\mathrm{A} \mathrm{pH}=12$ se consiguen partículas de morfología esférica. Además es posible obtener $100 \%$ cuprita haciendo la síntesis sin calentamiento obteniéndose partículas con morfología tetraédrica. 
En relación a la actividad antimicrobiana de las partículas de cuprita sintetizadas por ruta química empleando la glucosa como reductor en medio básico, se puede concluir que el óxido de cobre, cuprita, $\mathrm{Cu}_{2} \mathrm{O}$, tiene efecto antimicrobiano sobre la bacteria Staphylococcus aureus resistente a metilicina y que la Concentración Inhibitoria Mínima, CIM, corresponde a una concentración de $16 \mathrm{mg} / \mathrm{mL}$.

\section{AGRADECIMIENTO}

A la Facultad de Ciencias de la Universidad Nacional de Ingeniería, a la Pontificia Universidade Católica do Rio de Janeiro, PUC-Rio, Brasil, al laboratorio BISALAB y al Laboratorio de Microbiología de la Universidad Cayetano Heredia por el apoyo en los análisis realizados y al Vicerrectorado de Investigación de la Universidad Nacional de Ingeniería por el apoyo económico para la realización del presente Proyecto de Investigación.

\section{REFERENCIAS BIBLIOGRÁFICAS}

1. Zendejas-Manzo GS, Avalos-Flores H, Soto-Padilla MY. Microbiología general de Staphylococcus aureus: Generalidades, patogenicidad y métodos de identificación. Rev Biomed. 2014; 25:129-143.

2. Copper Connects Life. Cobre, salud, medio ambiente y nuevas tecnologías. [Internet]. Chile [citado el 15 mayo 2018]. Disponible en: http://www.umanda.cl/docs/Cobre Salud_Medio_Ambiente_Nuevas_Tecnologias.pdf

3. López-Fernández MJ, Llanos-Méndez A, Romero-Tabares A, Gómez RI. Eficacia, efectividad y seguridad de las superficies de cobre antimicrobiano en la prevención de las infecciones relacionadas con la asistencia sanitaria. [Internet]. Sevilla, España: Informes de Evaluación de Tecnologías Sanitarias AETSA; 2016. [citado el 11 abril 2018]. Disponible en: http://www.aetsa.org/download/publicaciones/07-A_AETSA_ Superficies-de-Cobre_DEF_NIPO.pdf

4. Flórez Palacios LM. Estudio de la actividad de las nanopartículas de óxido cúprico suspendido en pbs en cepas bacterianas estándar. [Tesis de Titulación]. Lima-Perú: Universidad Peruana Cayetano Heredia; 2017 [citado el 25 de octubre del 2017]. Disponible en http://repositorio.upch.edu.pe/handle/upch/462

5. Saikova SV, Vorob'ev SA, Nikolaeva RB, and Mikhlina YL, Conditions for the Formation of Copper Nanoparticles by Reduction of Copper (II) Ions with Hydrazine Hydrate Solutions. Russ J Gen Chem. 2010; 80 (6):1122-1127.

6. Obraztsova II, Simenyuk GY, and Eremenko NK. Preparation of Nanosized Copper Powderswith Controlled Dispersity. Russ J Appl Chem. 2010; 84 (6): 912-915.

7. Wang YJ, Zhou KG. Effect of $\mathrm{OH}-$ on morphology of $\mathrm{Cu} 2 \mathrm{O}$ particles prepared through reduction of $\mathrm{Cu}(\mathrm{II})$ by glucose. J Cent South Univ. 2012; 19: 2125-2129

8. Khan MA, Ullah M, Iqbal T, Mahmood H, Khan AA, Shafique M, et al. Surfactant Assisted Synthesis of Cuprous Oxide $\left(\mathrm{Cu}_{2} \mathrm{O}\right)$ Nanoparticles via Solvothermal Process. Nanosci Nanotechnol Res. 2015; 3 (1):16-22. 
9. Ismat Zerin L, Lutfun Naher H, Sarwaruddin Chowdhury AM, Gafur MA, Nuruzzaman K, Ruhul A. K. Preparation and Characterization of Copper Oxide Nanoparticles Synthesized via Chemical Precipitation Method. OAlib. 2015; 2: 1-8. doi: 10.4236/ oalib.1101409

10. Téllez MC, Rodríguez G, Reguera E. Síntesis de nanoestructuras de Oxido de cobre I y II. [Internet] III Simposio de Tecnología Avanzada, 17 al 24 de junio de 2009; México DF, México. [Citado 22 enero 2018] Disponible en: http://www.repositoriodigital.ipn. $\mathrm{mx} /$ bitstream/123456789/11513/1/libro3sta_34.pdf

11. Jadhav S, Gaikwad S, Nimse M, Rajbhoj A. Copper Oxide Nanoparticles: Synthesis, Characterization and Their Antibacterial Activity. J Clust Sci. 2011; 22 (2): 121-129.

12. Ahamed M, Alhadlaq HA, Khan MAM, Karuppiah P, Al-Dhabi NA. Synthesis, Characterization, and Antimicrobial Activity of Copper Oxide Nanoparticles. J Nanomater. 2014. Article ID 637858, 4 pages. doi: 10.1155/2014/637858.

13. Yi-Huang Hsueh, Ping-Han Tsai and Kuen-Song Lin, pH-Dependent Antimicrobial Properties of Copper Oxide Nanoparticles in Staphylococcus aureus. Int J Mol Sci. 2017;18(4). pii: E793. doi: 10.3390/ijms18040793

14. Yadav L, Tripathi RV, Prasad R, Pudake RM, Mittal J. Antibacterial Activity of Cu Nanoparticles against E. coli, Staphylococcus aureus and Pseudomonas aeruginosa. Nano Biomedic Eng. 2017; 9(1): 9-14. doi: 10.5101/nbe.v9i1.p9-14.

15. Cheng Ling S, Yuan Fang L, WenJing QI, Cheng Zhi W. Investigations of the interaction between cuprous oxide nanoparticles and Staphylococcus aureus. Sci China Ser B: Chem. 2009; 52(7): 1028-1032.

16. Ahmed MA, Mahmoud MH, Ashraf MES, Marwa RH. Antibacterial activity of cupric oxide nanoparticles against pathogenic bacteria. J Basic Environ Sci. 2016; 3:90-93.

17. Du BD, Phu DV, Quoc LA, Hien NQ. Synthesis and Investigation of Antimicrobial Activity of Cu2O Nanoparticles/Zeolite. J Nanopart. 2017. Article ID 7056864, 6 pages. doi: $10.1155 / 2017 / 7056864$

18. Liu KM, Takehiro Y, Kensuke K, Masazumi O. Preparation of $\mathrm{Cu}$ nanoparticles with ascorbic acid by aqueous solution reduction method. Trans Nonferrous Met Soc China. 2012: 2198-2203

19. Granata G, Yamaoka T, Pagnanelli F y Fuwa A. Study of the synthesis of copper nanoparticles: the roleof capping and kinetic towards control of particle sizeand stability. J Nanoparticle Res. 2016;18:133. doi: 10.1007/s11051-016-3438-6. 